\title{
Achieving Sharp Deliveries in Supply Chains through Variance Pool Allocation
}

\author{
Dinesh Garg, Y. Narahari, N. Viswanadham
}

\begin{abstract}
In this paper, our objective is to come up with a sound methodology to design supply chains with outstanding delivery performance. As the first step towards this objective, we consider supply chains with a linear workflow, which we call pipelined supply chains. We define a new index of delivery performance called delivery sharpness which measures the precision as well as the accuracy with which products are delivered to the customers. The specific problem we solve is: given the delivery sharpness to be achieved, how can we allocate variability across individual stages of the supply chain in a cost-effective way. We call this the variance pool allocation (VPA) problem. In formulating and solving the VPA problem, we explore interesting relationships among process capability indices $C_{p}, C_{p_{k}}$, and $C_{p_{m}}$, and generalize the notion of Motorola six sigma performance. The VPA problem leads to a four step design methodology and the resulting optimization problem is solved using the method of Lagrange multipliers. We present an interesting example of a supply chain in the plastics industry and illustrate the different steps of our methodology.

Keywords - Supply chain lead time, Cycle time compression, Delivery Probability (DP), Delivery Sharpness (DS), Process Capability Indices, Variance Pool Allocation (VPA), Generalized Motorola Six Sigma (GMoSS) Concept
\end{abstract}

\section{INTRODUCTION}

A $\mathrm{N}$ important design objective in supply chain networks is to achieve a high probability of delivery of finished products to the customer in a customer specified delivery window. This entails perfect synchronization among supply chain elements and individual business processes embedded within the supply chain process. This in turn requires variability reduction all along the supply chain. Variability reduction in supply chains is the subject of several research papers (several important references are listed in [1] and in [2]).

\section{A. Variance Pool Allocation Problem}

In this paper, we formulate and solve an important problem in the design of synchronized supply chains. We call this problem as the variance pool allocation problem (VPA). Given a supply chain and the mean

Computer Science and Automation, Indian Institute of Science, Bangalore - 560012 , India

Computer Science and Automation, Indian Institute of Science, Bangalore - 560 012, India

Mechanical and Production Engg, National University of Singapore, Singapore - 119260 and standard deviation of the end-to-end lead time for a certain product mix, the VPA problem seeks to optimally distribute the pool of variance among individual business processes so as to minimize cost and achieve outstanding delivery performance.

In this paper, we also describe the delivery performance of a supply chain in terms of two metrics. The first is a traditional metric, delivery probability (DP), which is the probability that a typical customer order is delivered during a customer-specified window. We show in the paper that the two popular process capability indices, $C_{p}$ and $C_{p_{k}}[3],[4]$ provide an appropriate vehicle for computing the delivery probability. The second metric is a new one that we propose, which we call delivery sharpness (DS), which is a measure of how close to the target (most desired) delivery date a customer order is actually delivered.

\section{B. Relevant Work}

The Motorola six sigma program for design tolerancing is described in [7], [8]. These reports also describe the process capability indices $C_{p}$ and $C_{p_{k}}$. These and other process capability indices are discussed in a comprehensive manner in [6], [4]. An attempt towards synchronizing the internal processes in a supply chain network for better delivery performance has been made by Narahari et al [9]. The authors emphasize the use of Motorola Six Sigma approach to analyze and design a given supply chain process for six sigma delivery performance. Two design problems are discussed in this paper: finding nominal pool and finding a variance pool. The present paper is a generalization of the above paper [9].

\section{Outline of the Paper}

Section 2 of this paper covers the description of process capability indices and relationship among each other, relationship with process yjeld and relationship DP as well as DS. The section also presents a generalization of the notion of Motorola six sigma quality. These findings are used in Section 3 to formulate the variance pool allocation problem for linear or pipelined supply chains. In Section 4, we present a four-step methodology for VPA in supply chains. Finally in Section 5, we describe a six stage supply chain in a plastics industry and apply our design methodology. 
TABLE I

Notation Used in the Definitions of PCIs

\begin{tabular}{|l|l|}
\hline$X$ & Lead Time or any quality characteristic. \\
\hline$\mu$ & Mean of $X$ \\
\hline$\sigma$ & Standard deviation of $X$ \\
\hline$L$ & Lower specification limit for $X$ \\
\hline$U$ & Upper specification limit for $X$ \\
\hline$\tau$ & Target value for lead time $X$, given by customer \\
\hline $\mathrm{T}$ & Tolerance for lead time $X$, given by customer \\
\hline$s$ & $|\tau-\mu|$ \\
\hline$p$ & $\min (|U-\mu|,|\mu-L|)$ \\
\hline
\end{tabular}

Implications and future work constitute Section 6 .

\section{Process Capability Indices: A New PERSPECTIVE}

\section{A. The Index $C_{p}$}

The process capability index, $C_{p}$, is defined as

$$
C_{p}=\frac{U S L-L S L}{6 \sigma}
$$

It is assumed later in our work that the distribution of $X$ is normal and the target value of lead time $\tau$ is the mid point of $U S L$ and $L S L$ for any business process. Hence $C_{p}$ can be expressed in following equivalent form.

$$
C_{p}=\frac{T}{3 \sigma}
$$

where $T=$ tolerance $=\frac{U S L-L S L}{2}$

$C_{p}$ measures only the potential of a business process, where by potential, we mean the probability of delivering the products within customer specified delivery window provided that $\tau=\mu$. This potential can be computed with the help of following relation:

$$
\begin{aligned}
\Phi\left(\frac{U-\mu}{\sigma}\right)-\Phi\left(\frac{L-\mu}{\sigma}\right) & =2\left(\Phi\left(\frac{T}{\sigma}\right)-0.5\right) \\
& =2 \Phi\left(3 C_{p}\right)-1
\end{aligned}
$$

where $\Phi(Z)$ is cumulative distribution function of standard normal distribution. This function is plotted in Figure 1.

B. The Index $C_{p_{k}}$

$C_{p_{k}}$ is defined as follows:

$$
C_{p_{k}}=\frac{\min (U S L-\mu, \mu-L S L)}{3 \sigma}=\left(\frac{p}{3 \sigma}\right)
$$

While $C_{p}$ measures the potential of the process, $C_{p_{k}}$ helps measure the actual yield (i.e. when $\tau \neq \mu$ ) and

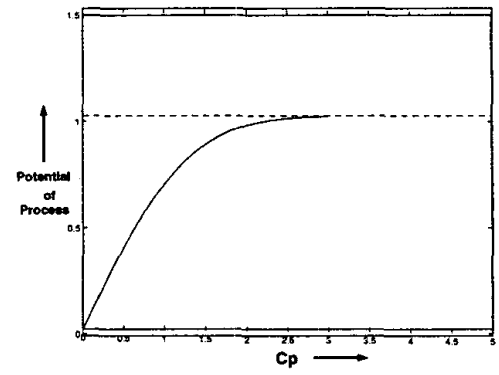

Fig. 1. Potential of the Process vs $C_{p}$

TABLE II

Formulae for Potential, Actual Yield, UPPER AND LOWER BOUND ON PROCESS YIELD

\begin{tabular}{|c|c|}
\hline Quantity & Formula \\
\hline Potential & $2 \Phi\left(3 C_{p}\right)-1$ \\
\hline Actual Yield & $\Phi\left(3 C_{p_{k}}\right)+\Phi\left(6 C_{p}-3 C_{p_{k}}\right)-1$ \\
\hline Upper Bound & $\Phi\left(3 C_{p_{k}}\right)$ \\
\hline Lower Bound & $2 \Phi\left(3 C_{p_{k}}\right)-1$ \\
\hline
\end{tabular}

actual yield of the process becomes equal to the potential of the process when $\tau=\mu$. potential of process $\geq$ actual yield of process.

In addition to potential and actual yield, we can define two other quantities: Upper Bound on process yield and Lower Bound on process yield. These bounds depend only on $C_{p_{k}}$ and the actual yield of the process lies between these two values. These four quantities represent areas of different regions under probability density function, $f_{X}(x)$, as shown in Figure 2. Table II summarizes the formulae for these four quantities. A proof of these formulae is provided in [10].
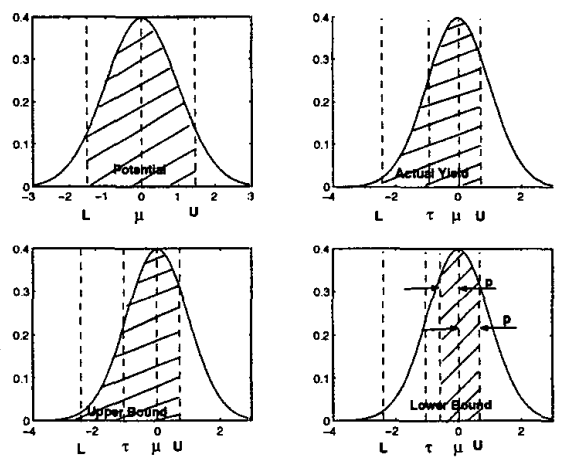

Fig. 2. Potential, Actual Yield, Upper and Lower Bound on Yield of the Process

\section{The Index $C_{p_{m}}$}

The index $C_{p_{m}}$ is defined as 


$$
C_{p_{m}}=\frac{\text { USL-LSL }}{6 \xi}=\frac{\mathrm{T}}{3 \sqrt{\sigma^{2}+s^{2}}}
$$

Quantity $E(L)=\sigma^{2}+s^{2}$ is known as "Expected Taguchi Loss" [6], [4].

D. Relationship and Dependencies among $C_{p}, C_{p_{k}}, C_{p_{m}}$

The following relations can be derived among $C_{p}, C_{p_{k}}, C_{p_{m}}[10]$.

$$
\begin{aligned}
C_{p} & \geq C_{p_{k}} \geq 0 ; C_{p} \geq C_{p_{m}} \geq 0 \\
C_{p_{k}} & =C_{p}(1-k) \\
\frac{1}{9 C_{p_{m}}^{2}} & =\frac{1}{9 C_{p}^{2}}+\left(1-\frac{C_{p_{k}}}{C_{p}}\right)^{2}
\end{aligned}
$$

where $k=\frac{s}{T}$.

Despite of mutual relationships among themselves, indices have a tight coupling with process yield also. It is easy to show [10] that for a given value of actual yield $\alpha$ (say), there exhibit lower and upper bounds for the values of both $C_{p}$ and $C_{p_{k}}$. We denote these lower and upper bounds by $C_{p}^{\alpha}, C_{p_{k}}^{\alpha}, \overline{C_{p}^{\alpha}}$ and $\overline{C_{p_{k}}^{\alpha}}$ respectively. A crisp idea behind the intent of these bounds is as follows:

If process's $C_{p}\left(C_{p_{k}}\right)$ is less than $C_{p}^{\alpha}\left(C_{p_{k}}^{\alpha}\right)$ then its actual yield can't be equal to $\alpha$; no matter how large $C_{p_{k}}\left(C_{p}\right)$ is. Similarly if process's $C_{p_{k}}$ is greater than or equal to $\overline{C_{p_{k}}^{\alpha}}$ then it's actual yield can't be less than $\alpha$; no matter how small $C_{p}$ is. The case with $\overline{C_{p}^{\alpha}}$ is little different. For any value of $C_{p}$ greater than or equal to $\overline{C_{p}^{\alpha}}$ it is possible to find a corresponding $C_{p_{k}}$ such that actual yield of the process is $\alpha$. Table III summarizes such bounds on $C_{p}$ and $C_{p_{k}}$.

\section{E. A Generalized View of Motorola Six Sigma Quality}

The basic idea of the Motorola Six Sigma concept $[7],[8]$ is that it identifies a "sigma $(\sigma)$ level" with each value of number of defects per million opportunities (npmo). In other words, a sigma level is attached with each value of actual yield of the process. As the actual yield increases the sigma level also increases. We call this sigma level as Delivery Probability (DP) because it essentially measures the upper bound of on time delivery probability in the context of the delivery process. This DP can be completely determined just by knowing $C_{p}$ and $C_{p_{k}}$.

Delivery Sharpness (DS): For any characteristic $X$ if we look at the definition of $C_{p_{m}}$, the increase in the variance decrease $C_{p_{m}}$ value. Similarly as mean $(\mu)$ comes closer to target $(\tau), C_{p_{m}}$ increases. Thus $C_{p_{m}}$ has as inherent feature to measure accuracy along with precision. Henceforth we call $C_{p_{m}}$ of the process as DS of the process.
TABLE III

Bounds on PCIS For ACtual YIELd $=\alpha$

\begin{tabular}{|c|c|}
\hline Bound & Formula \\
\hline$\frac{C_{p}^{\alpha}}{2}$ & $\frac{1}{3}\left(\Phi^{-1}\left(\frac{1+\alpha}{2}\right)\right)$ \\
\hline$\frac{C_{p_{k}}^{\alpha}}{3}$ & $\frac{1}{3}\left(\Phi^{-1}(\alpha)\right)$ \\
\hline$\overline{C_{p_{k}}^{\alpha}}$ & $\frac{1}{3}\left(\Phi^{-1}\left(\frac{1+\alpha}{2}\right)\right)$ \\
\hline$\overline{C_{p}^{\alpha}}$ & $\frac{1}{6}\left(3 \underline{C_{p_{k}}^{\alpha}}+\Phi^{-1}\left(1+\alpha-\Phi\left(3 C_{p_{k}}^{\alpha}\right)\right)\right)=\infty$ \\
\hline
\end{tabular}

In the situation like above, we define six sigma quality (or any other sigma) as the actual yield equal to $\left(1-3.4 \times 10^{-6}\right) \times 100 \%$ not the upper bound as per Motorola $6 \sigma$ program. Now our interest is to find out in how many different ways the bias and variance can be adjusted together without disturbing actual yield. This leads to a generalized view of six sigma quality, which we call GMoSS quality. In order to explain this idea let us start with equation:

$$
\text { actual yield }=\Phi\left(3 C_{p_{k}}\right)+\Phi\left(6 C_{p}-3 C_{p_{k}}\right)-1
$$

If we fix the value of actual yield as $\alpha$ in above equation then there will be two independent variables $C_{p}, C_{p_{k}}$, hence solution set will be unbounded. But already we have proved that for a given actual yield $\alpha C_{p}$ and $C_{p_{k}}$ are bounded within certain range. Hence the solution is bounded like: $C_{p_{k}}^{\alpha} \leq C_{p_{k}} \leq \overline{C_{p_{k}}^{\alpha}} ; \underline{C_{p}^{\alpha}} \leq C_{p} \leq \infty$.

If we substitute $\alpha=\left(1-3.4 \times 10^{-6}\right)$ and plot the curve, then all the points lying on the curve are $\left(C_{p_{k}}, C_{p}\right)$ pairs that correspond to the $6 \sigma$ quality level. This idea can be well understood with the help of Figure 3.

We can proceed one step further by looking at the connection between delivery probability and delivery sharpness in the light of the GMoSS notion. For this, we consider the plots of $\sigma$ quality levels on $C_{p_{k}}-C_{p}$ plane and then see how $C_{p_{m}}$ behaves on the same plot. For this purpose we use the identity relation among $C_{p}, C_{p_{k}}$ and $C_{p_{m}}$ (7) and plot this relation for a constant value of $C_{p_{m}}$ (say $C_{p_{m}}^{*}$ ). The curve comes out to be a part of hyperbola. From a process design point of view, it can be said that for a desired level of DS (i.e. $C_{p_{m}}$ ) and DP (i.e. $C_{p}, C_{p_{k}}$ ); this curve provides a set of 3-tuples $\left(C_{p}, C_{p_{k}}, C_{p_{m}}\right)$ which all satisfy these two 
requirements. The designer has to decide which one of the triples to choose depending upon other limitations. Figure 3 shows some contours of $C_{p_{m}}$ on $C_{p_{k}}-C_{p}$ plane.

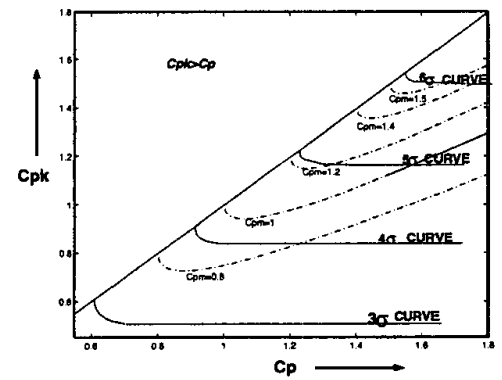

Fig. 3. Contours of $C_{p_{m}}$ on $C_{p_{k}}-C_{p}$ Plane

\section{Variance Pool Allocation (VPA) PROBLEM}

A. Linear Supply Chains: An Overview of Variation in Lead Time

Let us consider a linear or pipelined supply chain with $n$ processes as shown in Figure 4. In this supply

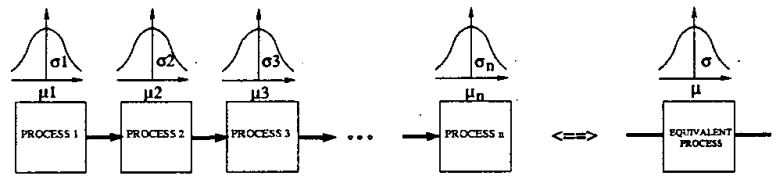

Fig. 4. A Pipelined Supply Chain Architecture

chain, material flows from process 1 to process $n$ and the end product is delivered to the end customer after processing at process $n$. The end-to-end lead time of each individual process (i.e. $X_{i}, i=1,2, \ldots, n$ ) is a continuous random variable.

\section{B. Assumptions on Nature of Business Process and Customer Delivery Window}

The design methodology proposed in this paper is based upon the following assumptions:

1. End-to-end lead time $X_{i}$ of each business process $i$ is normally distributed. Individual lead times are mutually independent of one another and also they are under statistical control.

2. There is no time elapsed between transforming the material from process $i$ to process $i+1$. Hence supply chain lead time $Y$ (which is equal to the total sojourn time of material within chain ) is equal to the sum of lead times of the individual processes which means $Y=\sum_{i=1}^{n} X_{i}$. It is easy to see that $Y$ will be normally distributed with $\mu=\sum_{i=1}^{n} \mu_{i}$ and $\sigma^{2}=\sum_{i=1}^{n} \sigma_{i}^{2}$ as it is sum of $n$ independent normally distributed random variables.

\section{Problem Description \\ C.1 Known Parameters}

1. Customer delivery window for lead time of each process i.e., $\left(\tau_{i}, T_{i}\right) \forall i$ and also for overall supply chain lead time i.e., $(\tau, T)$.

2. Mean $\mu_{i}$ of random variable $X_{i} \forall i$.

3. Cost of lead time per unit item produced, denoted by $C_{i}$, for each process $i$. This cost is that part of the total processing cost which is associated with lead time. For example if it is manufacturing process then it may be the opportunity cost of capital tied up with machinery. In most practical situations, cost of lead time per unit for any process $i$ increases as variance $\sigma_{i}$ decreases because $\mu_{i}$ is constant here.

It is known that $C_{p_{i}}$ is inversely proportional to $\sigma_{i}$, so any kind of function $C_{i}=f\left(\sigma_{i}\right)$ can be expressed in terms of $C_{p_{i}}$ which means $C_{i}=f\left(\sigma_{i}\right)=g\left(C_{p_{i}}\right)$. If underlying assumptions are assumed to hold, Taylor's theorem can be invoked on this function to approximate it, say, to a third order polynomial, in the following manner:

$$
C_{i}=a_{i 0}+a_{i 1} C_{p_{i}}+a_{i 2} C_{p_{i}}^{2}+a_{i 3} C_{p_{i}}^{3}
$$

Here $a_{i 0}, a_{i 1}, a_{i 2}, a_{i 3}$ are constants

\section{C.2 Decision Variables and Constraints}

In the VPA problem, the decision variables are variance values $\sigma_{i}$ of individual lead times $X_{i}$ of each process $i$ in the supply chain. Following are the constraints in the VPA problem:

1. DP for overall supply chain lead time should be at least at the level of $6 \sigma$ or any specified $\sigma$ level.

2. DS should be at least at the level of $C_{p_{m}}^{*}$.

\section{A Design Methodology for VPA Problem}

\section{A. Step 1: Problem Formulation}

The VPA problem can be expressed in the form of a nonlinear optimization problem as follows:

Objective Function:

Minimize

$C=\sum_{i=1}^{n} C_{i}=\sum_{i=1}^{n}\left(a_{i 0}+a_{i 1} C_{p_{i}}+a_{i 2} C_{p_{i}}^{2}+a_{i 3} C_{p_{i}}^{3}\right)$

Constraints:

1. DS for supply chain lead time $\geq C_{p_{m}}^{*}$

2. DP for supply chain lead time should be at least at the level of $6 \sigma$.

3. $C_{p_{i}} \geq 0 \forall i$ 


\section{B. Step 2: Formulation of Constraints in terms of De- cision Variables}

As per assumptions made in Subsection III-B, the variance of supply chain lead time $Y$ (i.e. $\sigma$ ) can be expressed in terms of variance $\left(\sigma_{i}\right)$ of processing time of process $X_{i}$. The following expression can be derived from it just by reengineering it.

$$
\frac{T^{2}}{9 C_{p}^{2}}=\frac{p^{2}}{9 C_{p_{k}}^{2}}=\frac{T_{1}^{2}}{9 C_{p_{1}}^{2}}+\frac{T_{2}^{2}}{9 C_{p_{2}}^{2}}+\ldots+\frac{T_{n}^{2}}{9 C_{p_{n}}^{2}}
$$

Equation (10) states that once the pair $\left(C_{p}, C_{p_{k}}\right)$ is chosen for supply chain lead time $Y$, the feasible solution set will get automatically fixed and it is the set of all those n-tuples $\left(C_{p_{1}}, C_{p_{2}}, \ldots, C_{p_{n}}\right)$ which satisfy this equation for the chosen value of $C_{p}$ and $C_{p_{k}}$.

The idea to get such a pair $\left(C_{p}, C_{p_{k}}\right)$ is to choose the pairs that satisfy both constraints (1) and (2) and use it in equation (10) in order to get the desired constraint in terms of decision variables.

\section{Step 3: Fixing Values for $C_{p}$ and $C_{p_{k}}$}

As pointed out in the last section, our problem is to find a $\left(C_{p}, C_{p_{k}}\right)$ pair which satisfies constraints (1) and (2) and also satisfies the relation $\frac{T^{2}}{9 C_{p}^{2}}=\frac{p^{2}}{9 C_{p_{k}}^{2}}$. This relation forces the desired $\left(C_{p}, C_{p_{k}}\right)$ pair to lie on the line $C_{p_{k}}=\frac{p}{T} C_{p}$ in $C_{p_{k}}-C_{p}$ plane. On the other hand, constraint (I) forces it to lie on or above the contour $C_{p_{m}}=C_{p_{m}}^{*}$ and constraint (2) forces it to lie on or above the $6 \sigma$ curve in the same plane. This will result in some feasible region on $C_{p_{k}}-C_{p}$ plane.Figure 5 shows different cases of such kinds of feasible regions depending upon relative positions of $C_{p_{m}}=C_{p_{m}}^{*}$ contour (in short $C_{p_{m}}$ curve) and $6 \sigma$ curve (in short $\sigma$ curve). From Figure 5 it is clear that feasible region in each case is the part of the line $C_{p_{k}}=\frac{p}{T} C_{p}$ that intersects with the shaded region. We have called it as line ' $O P$ ' 1 . In every case, the point ' $E$ ' where the line 'OP' enters into shaded region is taken as the final desired $\left(C_{p}, C_{p_{k}}\right)$ pair. The reason behind choosing this point is that if we choose any other point on the feasible region then the corresponding $C_{p}$ value will be high which will result in higher value of individual $C_{p_{i}}$ and hence higher delivery cost. Let us denote this pair as $\left(C_{p}^{*}, C_{p_{k}}^{*}\right)$.

\section{Step 4: Solving the Optimization Problem}

The underlying optimization problem can be rewritten as: Minimize cost $C$, given by equation (9), subjected to

\footnotetext{
${ }^{1}$ For the sake of clarity of picture, we have not shown the line 'OP' in all case except the case 1.
}

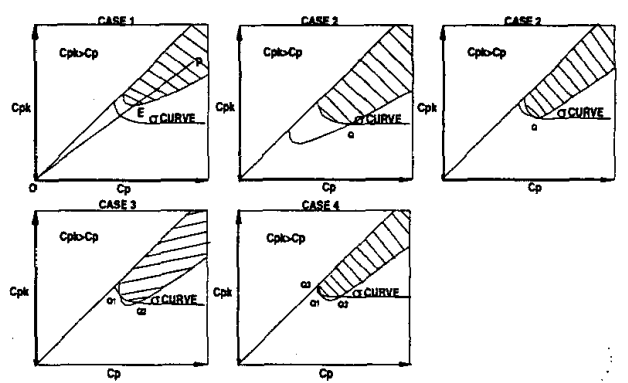

Fig. 5. All the possible configurations of $C_{p_{m}}$ curve, $\sigma$ curve and line 'OP' on $C_{p_{k}}-C_{p}$ Plane

$$
\begin{aligned}
\sum_{i=1}^{n} \frac{T_{i}^{2}}{C_{p_{i}}^{2}} & =\frac{T^{2}}{C_{p}^{*^{2}}}=\frac{p^{2}}{C_{p_{k}}^{*^{2}}} \\
C_{p_{i}} & \geq 0 \forall i
\end{aligned}
$$

The problem can be solved by using the method of Lagrange multipliers. The method is illustrated in the example in the next section.

\section{An ExAMPle}

Let us consider a supply chain for a plastics industry, as shown in Figure 6, to provide the basis for applying our design methodology. The supply chain has six business processes namely Procurement, Sheet Fabrication, Transportation, Manufacturing, Assembly, and Delivery. Let us assume that lead times (in Days) associated with these processes are represented by random variables $X_{i} ; i=1,2, \ldots, 6$ respectively and they satisfy the assumptions mentioned in subsection 4.2 .

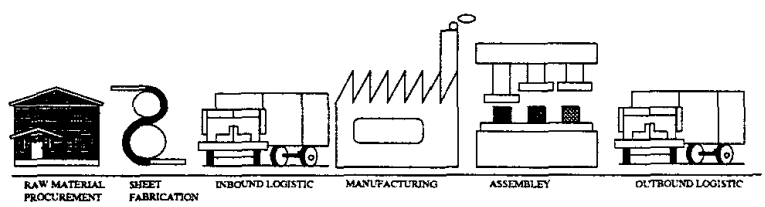

Fig. 6. An Example of a Pipelined Supply Chain: A Typical Plastic Industry Supply Chain

The problem here is to find out standard deviation $\sigma_{i}$ of lead time $X_{i}$ of all the processes in the chain such that a delivery probability of $6 \sigma$ level is attained and also a designated level of delivery sharpness, say, $C_{p_{m}}=1.42782$ (arbitrarily chosen in this case), is achieved. The known parameters are tabulated in $\mathrm{Ta}$ ble IV. Let the target value $\tau$ of the end-to-end supply chain lead time $Y$ be 82 days and tolerance $T$ be 6.5 days.

Steps 1 and 2: The objective here is to minimize the 
TABLE IV

Known Parameters for Plastic Industry Supply Chain Problem

\begin{tabular}{|c|c|c|c|c|c|c|c|}
\hline Process & $\mu_{i}$ & $\tau_{i}$ & $\bar{T}_{i}$ & $a_{i 0}$ & $a_{i 1}$ & $a_{i 2}$ & $a_{i 3}$ \\
\hline$X_{1}$ & 7 & 6 & 1 & 1 & 1 & 1 & 1 \\
\hline$X_{2}$ & 30 & 28 & 3 & 1 & 1 & 1 & 1 \\
\hline$X_{3}$ & 3 & 3 & 1 & 1 & 1 & 1 & 1 \\
\hline$X_{4}$ & 30 & 28 & 3 & 1 & 1 & 1 & 1 \\
\hline$X_{5}$ & 10 & 7 & 2 & 1 & 1 & 1 & 1 \\
\hline$X_{6}$ & 3 & 2.5 & 1 & 1 & 1 & 1 & 1 \\
\hline
\end{tabular}

following cost function:

$$
C=6+\sum_{i=1}^{6} C_{p_{i}}+\sum_{i=1}^{6} C_{p_{i}}^{2}+\sum_{i=1}^{6} C_{p_{i}}^{3}
$$

subjected to following constraints:

$$
\begin{aligned}
\sum_{i=1}^{6} \frac{T_{i}^{2}}{C_{p_{i}}^{2}} & =\frac{42.25}{C_{p}^{*^{2}}}=\frac{30.25}{C_{p_{k}}^{* 2}} \\
C_{p_{i}} & \geq 0 \forall i=1,2, \ldots, 6
\end{aligned}
$$

Step 3 If we draw the $6 \sigma$ curve, $C_{p_{m}}=C_{p_{m}}^{*}=1.42782$ curve and line $C_{p_{k}}=\left(\frac{5.5}{6.5}\right) C_{p}$ on $C_{p_{k}}-C_{p}$ plane then the situation will fall into case 2 of step 3 of our methodology. Solving the corresponding equations gives $C_{p}^{*}=1.89832236$ and $C_{p_{k}}^{*}=1.606272774$.

Step 4 Substitution of the values for $C_{p}^{*}, C_{p_{k}}^{*}$ will change the constraint (13) into $\sum_{i=1}^{6} \frac{T_{i}^{2}}{C_{p_{i}}^{2}}=11.724$. Now we will apply the Lagrange Multiplier Method in order to solve this optimization problem.

1. Lagrange Function Lagrange function $L\left(C_{p_{1}}, \ldots, C_{p_{6}}, \lambda\right)$ is given as:

$$
L\left(C_{p_{1}}, \ldots, C_{p_{6}}, \lambda\right)=C+\lambda\left(\sum_{i=1}^{6} \frac{T_{i}^{2}}{C_{p_{i}}^{2}}-11.724\right)
$$

2. Necessary Condition for Stationary Points Let point $\mathcal{P}^{*}=\left(C_{p_{1}}^{*}, \ldots, C_{p_{6}}^{*}, \lambda^{*}\right)$ correspond to the optimal point then this point must satisfy the following necessary conditions:

$$
\begin{aligned}
2 \lambda^{*} & =3 C_{p_{1}}^{*^{5}}+2 C_{p_{1}}^{*^{4}}+C_{p_{1}}^{*^{3}} \\
18 \lambda^{*} & =3 C_{p_{2}}^{*^{5}}+2 C_{p_{2}}^{*^{4}}+C_{p_{2}}^{*^{3}} \\
2 \lambda^{*} & =3 C_{p_{3}}^{*^{5}}+2 C_{p_{3}}^{*^{4}}+C_{p_{3}}^{*^{3}} \\
18 \lambda^{*} & =3 C_{p_{4}}^{*^{5}}+2 C_{p_{4}}^{*^{4}}+C_{p_{4}}^{*^{3}} \\
8 \lambda^{*} & =3 C_{p_{5}}^{*^{5}}+2 C_{p_{5}}^{*^{4}}+C_{p_{5}}^{*^{3}} \\
2 \lambda^{*} & =3 C_{p_{6}}^{*^{5}}+2 C_{p_{6}}^{*^{4}}+C_{p_{6}}^{*^{3}} \\
11.724 & =\frac{2}{C_{p_{1}}^{*^{2}}}+\frac{18}{C_{p_{2}}^{*^{2}}}+\frac{2}{C_{p_{3}}^{*^{2}}}+\frac{18}{C_{p_{4}}^{*^{2}}}+\frac{8}{C_{p_{5}}^{*^{2}}}+\frac{2}{C_{p_{6}}^{*^{2}}}
\end{aligned}
$$

Solving this system of equations by standard numerical methods we get only one real solution: $C_{p_{1}}^{*}=$ $C_{p_{3}}^{*}=C_{p_{6}}^{*}=1.005670 ; C_{p_{2}}^{*}=C_{p_{4}}^{*}=1.645700 ; C_{p_{5}}^{*}=$ $1.376280 ; \lambda^{*}=3.074458$. Under this operating condition cost of delivery $C^{*}$ is 38.601998 . It can be verified easily by the sufficiency condition that this point corresponds to the point of rninimum.

\section{IMPLICATIONS OF THE WORK}

In our view, this research has several important implications.

- It provides an elegant characterization for supply chain delivery performance in terms of two metrics, delivery probability and delivery sharpness, and three well known process capability indices.

- The above metrics and capability indices will provide a general framework for developing a design methodology for supply chains.

- The variance allocation problem is an important first step in the design of synchronized supply chains.

The work has some limitations. For example the analysis and design has been done only for make-toorder linear supply chains and that too for normally distributions lead times. Also, several assumptions have been made in formulating and solving the VPA problem. Many of these limitations can be surmounted in due course of time and provide important directions for further work.

\section{REFERENCES}

[1] N. Viswanadham, Analysis and Design of Manufacturing Enterprise, Kluwer Academic Publisher, 1999.

[2] S. Tayur, R. Ganeshan, and M. Magazine (Editors), Quan tative Models for Supply Chain Management, Kluwer Academic Publisher, 1999.

[3] Victor E. Kane, "Process capability indices.," Journal of Quality Technology, , no. 18, pp. 41-52, 1986.

[4] Samuel Kotz and Cynthia R. Lovelace, Process Capability Indices in Theory and Practice, Arnold, 1998.

[5] Russell A. Boyles, "The taguchi capability index.," Journal of Quality Technology, , no. 23, pp. 17-26, 1991.

[6] Madhav S. Phadke, Quality Engineering Using Robust Design, Prentice Hall PTR, Englewood Cliffs, New Jersey $07632,1989$.

[7] M. J. Harry, "The nature of six sigma quality," Tech. Rep., Motorola Inc., Motorola University Press, Schaumburg, II 60196-1097, 1997.

[8] M. J. Harry and R. Stewart, "Six sigma mechanical design tolerancing," Tech. Rep., Motorola Inc., Motorola University Press, Schaumburg, IL 60196-1097, 1988.

[9] Y. Narahari, N. Viswanadham, and R. Bhattacharya, "Design of synchronized supply chains: A six sigma tolerancing approach," San Francisco, April 2000, IEEE International Conference on Robotics and Automation, ICRA-2000.

[10] Dinesh Garg, Y. Narahari, and N. Viswanadham, "Achieving sharp deliveries in supply chains through variance pool allocation," Tech. Rep., e-Enterprises Lab, Indian Institute of Science, Bangalore, India, 2001. URL: http://lcm.csa.iisc.ernet.in/PSFILES/sharpdeliveries.ps. 